\title{
A Comparison of Frequency-Domain Block MIMO Transmission Systems
}

\author{
Reza Kalbasi, Member, IEEE, David D. Falconer, Life Fellow, IEEE, \\ Amir H. Banihashemi, Senior Member, IEEE, and Rui Dinis, Member, IEEE
}

\begin{abstract}
Block transmission techniques, with appropriate cyclic prefix and frequency-domain processing schemes, have been shown to be excellent candidates for digital transmission over severely time-dispersive channels, allowing good performance with implementation complexity that is much lower than traditional time-domain processing schemes. Orthogonal frequencydivision multiplexing (OFDM) modulation is the most popular block transmission technique. Single-carrier (SC) modulation using frequency-domain equalization (FDE) is an attractive alternative approach based on this principle. In this paper, we propose two new receiver structures for multiple-input-multiple-output (MIMO) channels employing SC (MIMO-SC) modulation and FDE schemes. These receivers have a hybrid structure with frequency-domain feedforward and time-domain feedback filters for intersymbol interference (ISI) and interference cancellation. The proposed schemes are compared with different MIMO systems employing OFDM modulation (MIMO-OFDM) receivers in terms of performance [bit error rate (BER) and throughput] and complexity. Our performance results show the superiority of MIMO-SC approaches relative to MIMO-OFDM in terms of the BER performance for the simulated scenarios. Also, the simulation results show that the proposed hybrid MIMO-SC receivers yield a higher throughput than a MIMO-OFDM system.
\end{abstract}

Index Terms-Bit error rate (BER), decision feedback equalizer (DFE), frequency-domain equalization (FDE), layered space-time (LST), multiple-input-multiple-output (MIMO) system, orthogonal frequency-division multiplexing (OFDM), single carrier (SC).

\section{INTRODUCTION}

$\mathbf{F}$ UTURE-GENERATION wireless systems are required to support a high quality of service at high data rates. For such high data rates, we can have severe time-dispersion effects with a very long intersymbol interference (ISI) span. In this case, the conventional time-domain equalization schemes are

Manuscript received February 11, 2006; revised January 29, 2007 and July 28, 2007. First published April 18, 2008; current version published January 16, 2009. An earlier version of this paper was presented at the Fifth IEEE Workshop on Signal Processing Advances in Wireless Communications, Lisbon, Portugal, July 11-14, 2004. The review of this paper was coordinated by Prof. M. Juntti.

R. Kalbasi is with ArrayComm LLC, San Jose, CA 95131 USA (e-mail: rkalbasi@arraycomm.com).

D. D. Falconer and A. H. Banihashemi are with the Broadband Communications and Wireless Systems Center, Department of Systems and Computer Engineering, Carleton University, Ottawa, ON K1S 5B6, Canada (e-mail: ddf@sce.carleton.ca; ahashemi@sce.carleton.ca).

R. Dinis is with the Institute for Systems and Robotics, Instituto Superior Técnico, Technical University of Lisbon, 1049-001 Lisbon, Portugal (e-mail: rdinis@ist.utl.pt).

Color versions of one or more of the figures in this paper are available online at http://ieeexplore.ieee.org.

Digital Object Identifier 10.1109/TVT.2008.923655 not practical since the signal processing requirements are very high. This can be more serious when conventional time-domain equalization methods are employed in high-data-rate multipleinput-multiple-output (MIMO) systems.

Cyclic prefix (CP)-assisted block transmission techniques employing frequency-domain equalization (FDE) schemes are known to be excellent candidates for severe time-dispersive channels, allowing good performance and implementation complexity that is much lower than those of traditional time-domain equalization techniques. Orthogonal frequency-division multiplexing (OFDM) [1] is the most popular frequency-domain technique. Single-carrier (SC) modulation using FDE techniques is another valuable candidate for highly dispersive channels in broadband wireless communications [2], [3]. In both cases, a CP is appended to each block, eliminating the interblock interference and converting the linear convolution that is associated with the channel into a circular convolution with respect to the useful part of the block. This allows lowcomplexity fast Fourier transform (FFT)-based receiver implementations. Although OFDM simplifies the equalization at the receiver, it has a high peak-to-average-power ratio and is more sensitive against the presence of frequency offset and phase noise [4]-[6].

It is well known that when we have deep frequency notches in the in-band region, the performance of linear equalizers can be very poor. In this case, it is better to employ a nonlinear equalizer, namely, a decision feedback equalizer (DFE), which presents a good performance/complexity tradeoff, provided that the error propagation is limited [7], [8]. In conventional DFEs, data is filtered by the feedforward filter symbol-by-symbol, and decisions are immediately fed back through the feedback filter to remove the postcursor ISI. Due to the delay in FFT processing, it is not practical to implement the feedback filter in the frequency domain. For this reason, a hybrid time-frequency SC-FDE was proposed in [2] and [9], employing a frequencydomain feedforward filter and a short time-domain feedback filter. The feedback filter is relatively simple to implement since it has only a small number of taps, and it can significantly outperform a linear FDE. An iterative DFE with frequencydomain feedforward and feedback has been proposed in [10] and [11]. Using iterative schemes, improved performance is achieved, whereas the complexity is increased.

Kadel [12] explored the possibility of combining the FDE with diversity techniques. Later, Clark [13] presented the potential of frequency-domain adaptive algorithms with diversity techniques. With the recent exploding interest in MIMO systems, MIMO equalizers have attracted significant 
attention [14]-[16]. However, MIMO channel equalization presents a significant challenge because MIMO receivers should simultaneously cancel ISI and cochannel interfering substreams. Time-domain MIMO equalizers have been introduced in [17]-[19].

In this paper, we present a comparison among various uncoded and coded frequency-domain block transmission systems (linear and nonlinear SC and OFDM receivers) with multiple antennas. First, we extend the ideas of Falconer et al. [2] to multiple antenna systems. The feedforward filter is implemented in the frequency domain and is designed to partially suppress ISI and interference, whereas the feedback signal is generated in the time domain to further remove the ISI and interference. The SC-FDE and OFDM receivers are compared based on two measures-bit error rate (BER) and throughput.

This paper is organized as follows. Section II describes the system model. In Section III, the different MIMO frequencydomain block transmission systems are presented. Section IV presents the signal processing complexity of the proposed techniques. The performance of the proposed receivers is evaluated in Section V. Finally, Section VI concludes this paper.

Notations: Lowercase (uppercase) fonts denote time (frequency) samples; bold fonts denote vectors and matrices; $(\cdot)^{T},(\cdot)^{*}$, and $(\cdot)(i, j)$ represent the transpose, the complexconjugate transpose, and the $(i, j)$ th entry of a matrix, respectively; $\mathbf{I}_{N}$ denotes the identity matrix of size $N ; \mathbf{e}_{i}$ denotes the $i$ th unit vector, and the vect $(\cdot)$ operator is defined as $\operatorname{vect}\left(\left[\mathbf{v}_{1}, \ldots, \mathbf{v}_{k}\right]\right)=\left[\mathbf{v}_{1}^{T}, \ldots, \mathbf{v}_{k}^{T}\right]^{T}$, where $\mathbf{v}_{1}, \ldots, \mathbf{v}_{k}$ are column vectors.

\section{SYSTEM MODEL}

We consider a discrete-time complex baseband model for an SC-MIMO system with $P$ transmit and $N$ receive antennas. The data is transmitted in blocks of $M$ data symbols $a_{p}(m)(m=0, \ldots, M-1)$ from the transmitter $p$ at a symbol rate $1 / T$. Each block is preceded by a $\mathrm{CP}$ to make the linear convolution of the channel equivalent to a circular convolution. At the receiver, the $\mathrm{CP}$ is discarded, and the received signals are sampled at $I / T$, where $I>1$ gives a fractionally spaced receiver whose performance is less sensitive to the sampling phase [20]. It is assumed that the channel is quasistationary; furthermore, perfect channel estimation and synchronization are assumed at the receiver. The $N$-dimensional received signal at the sampling instant $m$ can be expressed as

$$
\begin{array}{r}
\mathbf{r}(m)=\sum_{p=1}^{P} \sum_{k=0}^{M-1} \mathbf{h}_{p}(m T / I-k T) a_{p}(k)+\mathbf{n}(m T / I) \\
m=0, \ldots, M I-1
\end{array}
$$

where $\mathbf{r}(m)=\left[r_{1}(m), \ldots, r_{N}(m)\right]^{T}$, and $\mathbf{h}_{p}(m T / I-k T)=$ $\left[h_{p 1}(m T / I-k T), \ldots, h_{p N}(m T / I-k T)\right]^{T}(p=1, \ldots, P)$, with $h_{p n}$ denoting the sampled channel impulse response between transmitter $p$ and receiver $n . \mathbf{n}(\cdot)$ is the $N$-dimensional noise vector with mean zero and covariance matrix $\sigma^{2} \mathbf{I}_{N}$. The data symbols are assumed to be uncorrelated complex random variables, which are selected from a given constellation (e.g., a QPSK constellation). We define

$$
\begin{gathered}
A_{p}(l)=\sum_{m=0}^{M-1} a_{p}(m) \exp \left(\frac{-j 2 \pi m l}{M}\right) \\
l=0, \ldots, M-1 \\
\mathbf{N}(l+k M)=\sum_{m=0}^{M I-1} \mathbf{n}(m T / I) \exp \left(\frac{-j 2 \pi(l+k M) m}{M I}\right) \\
l=0, \ldots, M-1, k=0, \ldots, I-1 \\
\mathbf{H}_{p}(l+k M)=\sum_{m=0}^{M I-1} \mathbf{h}_{p}(m T / I) \exp \left(\frac{-j 2 \pi(l+k M) m}{M I}\right) \\
l=0, \ldots, M-1, k=0, \ldots, I-1 .
\end{gathered}
$$

After the FFT operation on a block of $M I$ samples of (1), the corresponding $M I$ frequency-domain samples can be expressed as

$$
\begin{aligned}
\mathbf{R}(l+k M)=\sum_{p=1}^{P} \mathbf{H}_{p}(l+k M) A_{p}(l)+\mathbf{N}(l+k M) \\
\quad l=0, \ldots, M-1, k=0, \ldots I-1
\end{aligned}
$$

If we define the $N I$-dimensional vectors $\mathbf{R}_{l}=\operatorname{vect}([\mathbf{R}(l)$, $\mathbf{R}(l+M), \ldots, \mathbf{R}(l+(I-1) M]) ; \mathbf{H}_{l}^{p}=\operatorname{vect}\left(\left[\mathbf{H}_{p}(l), \ldots, \mathbf{H}_{p}(l+\right.\right.$ $(I-1) M)])$; and $\mathbf{N}_{l}=\operatorname{vect}([\mathbf{N}(l), \ldots, \mathbf{N}(l+(I-1) M)])$, then (5) can be expressed as

$$
R_{l}=\sum_{p=1}^{P} \mathbf{H}_{l}^{p} A_{p}(l)+\mathbf{N}_{l}, \quad l=0, \ldots, M-1 .
$$

The transmitted symbols are assumed to have unit average power, i.e., $E\left[\left|a_{p}(m)\right|^{2}\right]=1$.

\section{HyBRID TIME-FREQUENCY RECEIVERS}

Here, hybrid time-frequency domain layered space-time (LST) DFE (LST-DFE) and hybrid time-frequency MIMODFE receivers are introduced. The hybrid time-frequency domain LST-DFE receiver for detecting the $P$ streams of data symbols, shown in Fig. 1(a), consists of $P$ successive multipleinput-single-output (MISO) hybrid time-frequency domain DFEs. At each stage, the "best" substream data block, in the MMSE sense, ${ }^{1}$ is selected, detected by a MISO-DFE, and transformed to frequency domain by an FFT operation. Then, this substream is subtracted from the received signal in the frequency domain, and the residual signal is passed to the next

\footnotetext{
${ }^{1}$ Since the complexity of computing the MMSE for all streams and selecting the best one for each stage is very high, a suboptimum scheme is used in our simulations. In this suboptimal approach, the streams are ordered and detected based on the first-stage MMSE. We observed that the suboptimum and optimum approaches have similar performance.
} 